\title{
Modo de proceder, tareas y elementos propios de una Universidad Católica ${ }^{1}$
}

\author{
Dr. Alberto Vásquez Tapia²
}

1. La historia de las universidades de occidente tiene más de mil años. La primera universidad de la cual tenemos noticia surge en la ciudad de Bolonia en Italia, en el siglo XI. Las universidades nacen para establecerse como espacios educativos dedicados a la generación, enseñanza y aplicación de todo conocimiento, incluidos los religiosos, los valores y las prácticas culturales sostenidas como válidas por una sociedad determinada. Estos propósitos se fueron concretando mediante el ejercicio del estudio de las letras, la enseñanza de los maestros a los discípulos y la difusión del saber que estas acciones iban generando dentro de sus recintos. En sus inicios la tarea intelectual primordial consistía en pensar y escribir sobre el hombre, la trascendencia, la religión, las artes y las humanidades.

2. La sociedad se instala como objeto de conocimiento de manera decisiva a partir del siglo XVI, el siglo del Renacimiento, de la Reforma de Europa, del cisma en la Iglesia
Católica, del Descubrimiento de América. Época caracterizada por la intensidad de los cambios, generadora de quiebres y desconcierto, pero también marcada por el esplendor creativo en las letras, en la filosofía, en las artes plásticas y en la espiritualidad cristiana. En ese ambiente la educación cristiana incide de modo decisivo en el mundo cultural, político e intelectual de la época, aportándole al humanismo renacentista, los elementos propios del humanismo cristiano. Los escritos de esa época están dedicados a abrazar "humanitas y fe", las letras y las virtudes, el rigor y la mansedumbre, el orden y el cuidado de la persona.

3. Recurriendo al lenguaje actualmente en uso se puede afirmar, entonces, que la tarea universitaria, por excelencia, desde siempre ha consistido en ser "constructores de humanidad", para lo que el ejercicio intelectual más preciado consiste en comprender los quiebres y las rupturas culturales ocurridos en

Trabajo recepcionado: octubre 2006

Trabajo aprobado: diciembre 2006

\footnotetext{
${ }^{1}$ Las ideas principales de este texto fueron presentadas en el XI Coloquio de Teología organizado por el Instituto Teológico de la Universidad Católica de Temuco.

${ }^{2}$ Sociólogo.
} 
un momento histórico determinado, de modo de devolver al hombre el pleno sentido de la vida humana y potenciar, así, su capacidad de constructor de su cultura, de ser autor de la historia. En los ambientes creyentes, esta tarea se realiza mejor y se perfecciona cuando se la verifica en una estrecha relación entre fe y cultura, puesto que el sentido más pleno de la vida humana habita en la relación entre el hombre y su Creador.

4. En el transcurso de esta milenaria tarea educativa, el ejercicio honesto y agudo de la crítica como herramienta intelectual y la disonancia inteligente y argumentada, se transforman en valores organizacionales aceptados, deseados y cultivados. Ese ejercicio crítico genera tensiones tanto al interior, como en la relación de la universidad y su entorno. En consecuencia, habría que partir por aceptar que la disonancia, las disputas, y hasta las tensiones que producen, son consustanciales a la universidad. Las universidades son espacios críticos. Asimismo, debe aceptarse que toda universidad es siempre un proyecto, está permanentemente en construcción, no vive de certezas definitivas, vive de preguntas. Estas condiciones organizacionales de base producen desasosiegos, tensiones, crisis. Esos efectos, las tensiones y las crisis, son por lo tanto, parte de su modo de ser y de hacer. Efectivamente, en todas las épocas y lugares se pueden constatar fuertes tensiones al interior de las universidades, y entre éstas y los grupos predominantes de su sociedad contemporánea.

5. Al interior de los claustros, una de las tensiones más profundas radica en la permanente tensión entre ciencia y conciencia, entre el conocimiento y la ética, más propiamente entre el método y la ética. La producción intelectual en el mundo universitario resulta legítima en la medida que verifica y perfecciona su capacidad para ofrecer, desde su saber, respuestas válidas a las preguntas que se instalan en la frontera de sus campos epistemológicos. No obstante, esta sola enunciación no resulta suficiente. La discusión se juega en la disputa acerca de la aceptación o no de una frontera del saber definida por límites del orden de la ética. Esta discusión acompaña la vida universitaria y las tesis en disputa se suceden a lo largo de los años. En esta frontera existen posturas contrarias, en algunos casos hasta contradictorias.

6. En determinados ambientes académicos, la ciencia parece ser considerada como un fin en si misma y con una finalidad autoreferida. Los saberes y las disciplinas son cultivados sin más límite que los procedimientos aceptados en los marcos epistemológicos y metodológicos dentro de los cuales producen sus expertos. En otros ambientes al conocimiento y la ciencia se les confiere la condición de medios. En éstos, a la producción intelectual, artística, científica y técnica -también validada por cánones y marcos metodológicos-, se le exige arrancar explícitamente desde una determinada concepción de hombre y de sociedad y fundamentarse en una teleología determinada, esto es, los impactos y las consecuencias de sus productos y resultados. Generalmente, pero no exclusivamente, esta exigencia al científico resulta más clara y marcada en las universidades creyentes. En el caso de las universidades católicas, el horizonte ético desde el cual se concreta dicha exigencia está dado por el humanismo cristiano.

7. La autonomía institucional y la libertad de cátedra del sujeto académico para ejercer la crítica, buscar la verdad y mejorar la eficacia científica son rasgos distintivos propios de la impronta universitaria y resultan medulares en la construcción identitaria de estas casas de estudios. Esto no está en cuestión. Lo que está en el tapete es el imperativo que tiene toda universidad de declarar los criterios mediante los cuales logra, o busca lograr, la mejor ecuación posible entre ciencia y valores, entre el desarrollo científico y un determinado horizonte ético, al cual adhiere. Ninguna universidad se desentiende de esta permanente búsqueda, sea ésta católica o no católica. Renunciar a esa búsqueda sería simplemente renunciar a su condición de universidad. Hoy, se sabe con claridad que no existe la pretendida neutralidad de las ciencias. 
8. Fuera de los claustros, en la relación universidad y sociedad, se sitúan los desafíos y cuestionamientos más importantes y valiosos para las universidades dedicadas a la función de ser constructoras de humanidad, constructoras de cultura. En escenarios sociales caracterizados por proceso de transformaciones culturales, la tarea que emerge en la relación universidad y sociedad se torna especial y particularmente desafiante, rica y útil para la producción intelectual.

9. En los días actuales, las universidades experimentan una situación análoga a la vivida en el Renacimiento y la Reforma Europea del siglo XVI. En el actual momento histórico, una vez más se asiste a cambios radicales en los escenarios científicos, tecnológicos, económicos y culturales. Estamos ante un cambio de época, que afecta y altera los modos de entender, de valorar y de hacer en todos los órdenes de la actividad humana. Cambios que hacen tambalear las referencias básicas aprendidas sobre la vida, en medio de un ambiente que socava el sentido de la relación de la persona con los demás, con el trabajo, con la misma naturaleza y la cultura. El Concilio Vaticano II habla de « una nueva era de la historia de la humanidad. ${ }^{3}$ La Iglesia invita a vivir este tiempo como kairós, es decir, como un tiempo favorable para una renovada verificación del acontecer vivificador de Dios en J esucristo; tiempo en el que "Ios nuevos rasgos de la cultura constituyen otros tantos desafíos y puntos de apoyo". 4

10. El desafío más decisivo radica en una búsqueda incesante y tesonera del ser humano por hacerse cargo de su existencia. Se trata de una vieja promesa de emancipación, que hunde sus raíces en el movimiento cultural de la ilustración. 5 "Sectores crecientes de la humanidad buscan incansablemente hacerse cargo de su propia existencia mediante el uso de la razón, de la capacidad de pensar y de decidir por sí mismo, mediante el dominio del mundo y de la vida a través del saber científico, del desarrollo tecnológico y del trabajo humano. Esa promesa y esa búsqueda, como toda búsqueda del ser humano, están cruzadas por tensiones y desafíos propios de su radical ambigüedad". 6

11. La tarea está bien planteada en la Constitución Apostólica de Juan Pablo II, Ex Corde Ecclesiae, y en el texto Pontificio "Para una Pastoral de la Cultura". En éstos, el reto consiste en encontrar una articulación fundada entre la autonomía plena de la razón humana y el reconocimiento ineludible de sus límites; "tanto de los límites que le impone la objetividad misma del ser humano y de la naturaleza, como de los límites que provienen de la propia incapacidad de la razón para darse un fundamento absoluto. De este modo, articular autonomía racional y ofrenda de la razón; conquista y gratuidad; autonomía y obediencia es quizá el desafío más hondo de la razón moderna de cara a un nuevo humanismo".8 Las universidades católicas tienen aquí un desafío exigente, una tarea inconclusa, un examen aún no aprobado.

12. Otra tarea tiene relación con la pertinencia social del trabajo universitario. En estos años, se hace clara la necesidad de estrechar la distancia entre lo que se enseña y las necesidades que experimentan las personas, las ciudades, las organizaciones, el contexto que rodea el centro universitario. En muchas universidades, los programas son repeticiones de modelos que se enseñan y funcionan en el primer mundo. La aplicabilidad y la pertinencia de lo enseñado no resulta clara, los servicios demandados que no cuentan con recursos resultan casi imposible ofrecerlos, acentuándose así, una especie de divorcio entre la universidad y la realidad de las personas comunes, especialmente de los grupos y sectores sociales que no cuentan con medios de financiamiento.

\footnotetext{
${ }^{3}$ Gaudium et Spes, N ${ }^{\circ} 54$

${ }^{4}$ Para una pastoral de la cultura, $\mathrm{N}^{\circ} 7$

${ }^{5}$ KANT, Immanuel, ¿Qué es la llustración?, 1784

6 López, Francisco: La Universidad para un Nuevo Humanismo". Departamento de Ciencias Sociales, Universidad Alberto Hurtado

${ }^{7}$ Consejo Pontificio de la Cultura, para una Pastoral de la Cultura, 4 de junio de 1999, №22.

8 López, Francisco: La Universidad para un Nuevo Humanismo". Dpto de Ciencias Sociales, Universidad Alberto Hurtado.
} 


\section{Revista CUHSO volumen 12 n 2}

\section{Universidad Católica}

13. La universidad católica es universidad como cualquier otra, pero admite para sí misma, para su institución y sus sujetos académicos, las tareas y los desafíos anteriormente aludidos, que emanan del hecho de adherir a una antropología cristiana, una visión cristocéntrica de la existencia y a una teleología definida en el marco del horizonte ético cristiano. Esta adhesión debería distinguir y cualificar su producción académica, su servicio al país y su interlocución pública. Para muchos académicos, la misma inspiración cristiana pareciera ser entendida como un factor negativo, en la medida en que condicionaría en cierto modo la autonomía, la libertad de docencia y de investigación. El cuestionamiento está lejos de ser bizantino: ¿Cómo conjugar autonomía y libertad con la sujeción a declaraciones de principios y cartas magnas, por añadidura de carácter confesional? ¿Constituirá el carácter "católico" una limitación al pensamiento y a la investigación? ¿Cómo ubicarse frente a otras universidades públicas o privadas que no tienen tales desafíos? ¿Qué distingue a una universidad católica de otras que no se proclaman como tales? ¿En qué se diferencia su oferta? ${ }^{9}$

14. En noviembre del 1997, en el Sínodo de América, el P. Peter-Hans Kolvenbach ${ }^{10}$, transmitió una interrogante candente: "la universidad católica sigue preocupada hasta hoy con el temor de que pudiera resultar verdadera la afirmación de George Bernard Shaw de que una universidad católica es una contradicción en términos. La contradicción es insoluble si se la plantea en términos de un dilema: o universidad o católica". La resolución de la tensión consistiría en conjugar ambos extremos, considerándolos como dos aspectos de una misma institución, no en términos contradictorios, sino complementarios.

15. El sustantivo universidad indica que las bases del modelo de la universidad católica son primariamente intelectuales y que, como toda universidad, está Ilamada por vocación y tradición a constituirse en auténtico espacio destinado a la generación plural de las ideas, a la formación de sujetos autores de la historia, al desarrollo de la ciencia y del humanismo. Un espacio para la generación y aplicación del conocimiento, también un espacio dedicado a devolverle el señorío al hombre sobre la cultura. Un espacio de estudio riguroso, serio, basado en el diálogo y la reflexión. Un lugar donde los intelectuales se dedican a generar propuestas eficaces para influir en la formación de una sociedad mejor, más próspera y más justa.

16. El calificativo de católica significa que a la ciencia y al conocimiento se les confiere la condición simplemente de medio, en consecuencia los sujetos académicos que las cultivan aceptan y adhieren al desafío de cualificar su producción intelectual, artística, científica y técnica - ciertamente en acuerdo con los estándares metodológicos de las respectivas ciencias-, desde una visión cristiana del hombre y del mundo y dirigida con claridad hacia finalidades éticamente aceptables y deseables en el horizonte ético cristiano, esto es, un estilo específico de relacionar al hombre consigo mismo, con los demás y con lo creado.

17. En el plano docente, la universidad católica adopta un estilo propio de concebir la relación entre enseñanza y aprendizaje, para acompañar en libertad y responsabilidad al estudiante en su proceso de descubrimiento, construcción y conquista de las verdades del saber, del saber ser y del saber hacer. Un estilo pedagógico que arranque y considere el contexto como parte integrante y hacia el cual se dirigen los esfuerzos formativos. Un estilo caracterizado por marcar a los estudiantes mediante la propuesta de experiencias profundas y significativas de contacto con la realidad, con los demás y con Dios. Un proceso de enseñanza que destina tiempo a la reflexión,

\footnotetext{
${ }^{9}$ Codina, Gabriel: “Los elementos del modelo universitario ignaciano". En Vásquez, Alberto: Reflexiones para un Modelo Universitario. ITESO, Guadalajara, México, 1998.

${ }^{10}$ Idem
} 
que impulsa al estudiante a tomar decisiones propias y fundamentadas, que lo llevan a actuar y hacerse cargo de las consecuencias de sus decisiones y acciones. En otras palabras, una formación integral del estudiante para formarle el talante que le de sentido ético y social al uso de sus talentos.

\section{Necesidad de diálogo y pluralismo}

18. La posición consistente en considerar la dimensión ética cristiana como parte integrante de la producción intelectual, necesita ser correctamente dialogada con quienes piensan distinto. En los ámbitos universitarios, este tema debe ser parte de un serio análisis, para que logre ser comprendido, aceptado y respetado. A modo de ejemplo de este tipo de diálogo intelectual entre creyentes y agnósticos, menciono el intercambio de cartas que sostuvieron en una revista italiana el Cardenal Carlo Maria Martini y el escritor Umberto Eco. ${ }^{11}$

19. El diálogo es la manera más eficaz de construir una auténtica universidad y dotarla de catolicidad. A la universidad católica debe caracterizarla un clima de respeto y pluralismo entre sus académicos y sus estudiantes. Las modalidades reflexivas y el tiempo que se destine al diálogo, constituyen formas eficaces para tender un puente entre la fe y la ciencia, entre la ciencia y el humanismo. Mediante el diálogo, se pueden abordar de un modo razonable las complejidades de ser universidad y católica en la sociedad moderna. Dialogar permite la inclusión en logro de un "animus societatis", en la construcción de la comunidad y en el logro de la misión universitaria.

20. La cuestión a dilucidar es qué se entiende por diálogo y por pluralismo. Diálogo no es una simple conversación, proviene del vocablo logos que significa palabra y del vocablo dia que significa entre, es decir, diálogo es interacción verbal entre personas en la cual se intercambian ideas (pensamientos) y afectos (emociones). "Mediante el diálogo se Ilega a conocer a otra persona y, por ende, a uno mismo. Un diálogo profundo, cristiana y racionalmente inspirado, supone aceptar al otro desde su alteridad como un interlocutor válido, de igual valor y dignidad" ${ }^{12}$. El diálogo supone ordenar los afectos y pensamientos en función de una recta intención, pero asimismo supone creer sinceramente que el otro también argumenta movido por una recta intención. Sin este factor de confianza entre los interlocutores, no existen condiciones de posibilidad para establecer un diálogo. En esta definición el diálogo no es una simple conversación. "En una conversación el eje es el tema, en el diálogo el tema son las personas. ${ }^{13}$ La conversación requiere de capacidades lingüísticas y expresivas, el diálogo exige entrega de la persona. Una conversación no compromete a los interlocutores, el diálogo deja huellas en los participantes".

21. Por otro lado, pluralismo supone la existencia de cosmovisiones, creencias y valores diferentes. Los credos de unos pueden ser coincidentes o diferentes con los credos de otros, pueden incluso parecer contrarios y llegar a ser hasta contradictorios. Los credos profesados en el mundo universitario pueden pertenecer al orden de las ciencias, de las religiones o de las ideologías. Ciertamente, lo propio del ambiente universitario es la expresión de una diversidad de credos. La diversidad nutre la producción intelectual.

22. ¿Cómo establecer un ambiente de diálogo pluralista en una universidad y católica? En primer lugar, es básico afirmar que la fe no es una pura paradoja, ni se agota en el rito religioso. "La fe sólo en cuanto intelectualmente razonable es digna de Dios y del hombre: la fe no teme la razón, sino que la busca y confía en ella"14. En segundo lugar, las personas que dialogan deben estar dispuestas a explicitar y testimoniar su credo y a actuar

\footnotetext{
${ }^{11}$ Ecco, Umberto y Martini, Carlo Maria. ¿En qué creen los que no creen?. Taurus, México, 1997.

12 Informe ETHOS № 8. Centro de Ética, Universidad Alberto Hurtado, Santiago de Chile, 2000.

13 Idem.

${ }^{14}$ La universidad por un nuevo humanismo: El jubileo de los universitarios. Congregación para la Educación Católica, Roma, 2000.
} 
en coherencia con sus creencias y valores. En otras palabras, para dialogar se requiere de sujetos dispuestos a explicitar sus creencias, no existe pluralismo en el silencio o en la omisión. A veces, en nombre de un supuesto recato en favor del pluralismo se calla la dimensión ética cristiana de la universidad. El pluralismo es una dimensión en construcción permanente, precisa de hombres y mujeres dispuestos a decir su verdad. Personas que aceptan la parcialidad de su propia verdad, de sus racionalidades y visiones, que tienen en común la búsqueda de la verdad.

\section{Hacia el modo universitario de proceder}

23. La construcción de la catolicidad de un proyecto universitario se puede entender, también, como la construcción de un modo de ser y de proceder en el ámbito propio del espacio universitario. Ese "modo de ser universidad" supone, en primer lugar, la presencia de una condición de base: la misión institucional y sus finalidades son asumidaslibre y deliberadamente por los sujetos académicos. Por lo tanto, dicha misión, los valores que privilegia y las finalidades de las que se ocupa deben estar declaradas, de modo que quien Ilega o quien pertenece a su comunidad pueda ejercer su derecho a decidir libremente su adhesión y se le permita presentar los modos personales mediante los cuales contribuye, desde sus prácticas académicas y relacionales, al logro de dicha misión institucional.

24. En segundo lugar, para la conformación de ese modo de ser y de proceder, se debe reconocer que las universidades católicas tienen en común con las demás universidades características tales como: a) el cultivo de las ciencias y las artes, mediante la generación, depuración, transmisión y aplicación del conocimiento; b) la difusión pública del pensamiento y una relación pertinente con su entorno; c) la libertad de cátedra: d) la formación de profesionales; e) la búsqueda de la excelencia. En adición a estas características, las instituciones católicas tienen cinco notas distintivas: 1) la comprensión de las ciencias como medios, por lo tanto, la aceptación de límites éticos a los modos de operar con el conocimiento, en el horizonte del humanismo cristiano; 2) la formación del estudiante concebida como la formación de todo el hombre y toda la mujer; 3) un fuerte compromiso social enmarcado en la opción por los más pobres; 4) la presencia de espacios académicos y manifestaciones propias de la fe y la inspiración cristiana que profesa; y 5) el cuidado personal por cada uno y la atención puesta en cuidar la vida comunitaria.

25. La tarea consistiría, entonces, en aclarar satisfactoriamente de qué manera cada una de las notas específicas de la catolicidad se realiza en relación con cada una de las características de toda universidad $^{15}$. En otras palabras, el modelo puede visualizarse como un esquema matricial, que tendría en uno de sus ejes lo común de toda universidad (sustantivo) y en el otro lo propio de la catolicidad (especificidad). El perfil del "modo de proceder" y sus consecuencias prácticas, aparecería mediante los cruces y conexiones de ambos ejes. Realizar esta tarea debería ser un ejercicio colectivo, informado y reflexivo de todos los actores universitarios. Importa mucho más ese proceso de diálogo, disputas y reflexión, que los textos que finalmente se logren redactar.

26. Las tesis en disputas y las discusiones que nortearan la búsqueda de ese modo de ser universidad y católica, probablemente harán emerger con mayor claridad la existencia de diversas lógicas en los ambientes universitarios. Al menos se pueden declarar tres lógicas mayores: la lógica académica, la lógica organizacional y la lógica apostólica. La "lógica académica" determina los modos mediante los cuales una comunidad de profesores, organizada por área de conocimiento, toma decisiones acerca de las prácticas académicas a ser desarrolladas en el ámbito de la investigación, la docencia y la

\footnotetext{
${ }^{13}$ Vásquez, Alberto: Hacia un Modelo Universitario Ignaciano. Documento de trabajo de la Comisión para el Modelo del Sistema UIA-ITESO, México, 1996
} 
extensión, basadas en losparámetros y cánones epistemológicos y metodológicos propios de las disciplinas que cultiva, procurando satisfacer determinadas finalidades. La mirada central es más marcadamente departamental.

27. Por otro lado, la universidad tiene también una "lógica organizacional" de la cual se ocupan determinados sujetos responsables de los sistemas y mecanismos de planeación, articulación y evaluación. El gobierno y la gestión de la organización universitaria tienen también una racionalidad a la base que condiciona las decisiones, en función de objetivos declarados. Por ej emplo, la organización debe ser eficiente, productiva, viable, financiada.

28. En las universidades católicas se agrega, al menos, una tercera lógica, la "lógica apostólica". En ésta, la misma universidad es simplemente un medio. Los Consejos Pontificios, Episcopales y Congregacionales, desde sus horizontes éticos, religiosos y espirituales analizan los signos de los tiempos, disciernen y toman decisiones relativas a los énfasis que desean impregnar en sus obras educativas. La educación, la universidad, la escuela son un medio para evangelizar la cultura, para construir una mejor humanidad.

29. Estas lógicas no son siempre coincidente. Tampoco puede afirmarse que sean contradictorias. La hipótesis de base es que actualmente existen más similitudes que elementos mutuamente excluyentes entre ellas. En general, cuando la misión y los valores profesados y practicados están orientados rectamente hacia la búsqueda de las verdades profundas que subyacen en la vida, en la ciencia, en el hombre, estas lógicas y sus actores se potencian y emergen grandes posibilidades de articularlas en una universidad católica.

30. En consecuencia, se está afirmando que estas lógicas son constitutivas del modo de ser de una universidad y católica. También es cierto que integrarlas y traducirlas en procesos de institucionalización muchas veces genera tensiones y que su debate genera tesis en disputas. Pero estas manifestaciones son positivas, son parte de la vida y de la historia de las universidades. Lo que no debe ocurrir es imponer una lógica encima de la otra. Tampoco debe una universidad omitirse al debate manteniéndolas en líneas paralelas. Cuando esto ocurre, muere el proyecto de lograr ser universidad y católica.

31. En resumen, en las universidades católicas se debe establecer como dato de entrada la convicción de que las ciencias, sus mediaciones metodológicas y sus resultados están subordinados al horizonte ético cristiano, colocando a las ciencias y a la misma universidad como medios para servir y a hacer a todos los hombre y mujeres más personas, más dignos, más plenos. En segundo lugar, el diálogo y el respeto por las diversas visiones son parte del modo de proceder de estas casas de estudios. En tercer lugar, que este modo de proceder emerge cuando se busca reconocer las prácticas congruentes que resultan al entrecruzar las dimensiones que corresponde a lo común y sustantivo en toda universidad, con las notas específicas de la cualidad de católica. En cuarto lugar, que esa intersección está contenida en un espacio organizacional, por lo tanto en éste conviven, no sin tensiones, diversas lógicas que son constitutivas, que deben ser respetadas y que no deben anularse.

32. Finalizo resaltando mi convicción que nuestro modo de ser universidad, surge cuando la apuesta está en la persona, en la confianza, en la libertad, en la construcción reflexiva y pluralista. Noscorresponde colaborar activamente para generar condiciones de posibilidad para lograr esa universidad, mediante una búsqueda intencionada y apasionada de la verdad. Estamos nuevamente en una época en que el hombre y la mujer precisa recuperar la capacidad de encontrar el sentido profundo de la vida. Los universitarios, todos, estamos desafiados a ser constructores de esa nueva humanidad. Los universitarios católicos lo buscamos mediante la integración entre la fe y la ciencia. Pero a todos nos une 
Revista CUHSO volumen $12 \mathrm{n} \div 2$

un humanismo auténtico. Para algunos será el servir al hombre, su dignidad y su bienestar. Para otros, es servir a ese mismo hombre porque es el hijo predilecto y amado de Dios.

\section{BIBLIOGRAFÍA}

AUSJ AL (1995) Desafíos de América Latina y propuestas educativas.

CONGREGACIÓN GENERAL 34 (1995) Compañía de J esus: Los jesuitas y la vida universitaria.

ICAJE (1986) Características de la educación de la Compañía de J esús.

ICAJE (1993) Pedagogía Ignaciana: un planteamiento práctico.

ICAJE (1993) Pedagogía ignaciana hoy, Peter Hans Kolvenbach, S.J.

O'KEEFE, J OSEPH M. SJ (1997) Catholic Higher Education at the Turn of the New Century. Center for International Higher Education, School of Education, Boston College.

TURNER, FRANK M., ED. (1996) The Idea of a University. J ohn Henry Newman. Yale University Press, London.

TRIPOLE, MARTIN, ED. (1999) Promised Renewed. J esuit Higher Education for a New Millenium. Loyola Press, Chicago.

VÁSQUEZ, ALBERTO (1997) Reflexiones a diez años de las características de la educación de la Compañía de Jesús. ITESO, Guadalajara, México.

VÁSQUEZ ALBERTO (1998) Reflexiones para un modelo educativo universitario; ITESO, Guadalajara, México.

WORLD BANK: HIGHER EDUCATION IN DEVELOPING COUNTRIES (2000) Peril and Promise. The Task Force on Higher Education and Society. Washington. 